ISSN: 2320-7167

Volume 10 Issue 01 January 2022, Page no. - 2540-2545

Index Copernicus ICV: 57.55, Impact Factor: 7.184

\title{
Co-Homological Dimension of Random Graph Groups
}

\author{
Abdel Radi Abdel Rahman Abdel Gadir Abdel Rahman', Hayat Ali Aboelkeram Elzain ${ }^{2}$, Adam Osman Ali \\ Mohammed $^{3}$, Subhi Abdalazim Aljily Osman ${ }^{4}$ and Neama Yahia Mohamed ${ }^{5}$ \\ ${ }^{1,2}$ Department of Mathematics, Faculty of Education Omdurman Islamic University, Omdurman, Sudan \\ ${ }^{3}$ Department of Mathematics, Faculty of Engineering, Sennar University, Sennar, Sudan \\ ${ }^{4}$ Department of Mathematics, Faculty of Computer Science and Information Technology, University of ALBUTANA, Sudan \\ ${ }^{5}$ Department of Mathematics, College of Science, Tabuk University, Tabuk, Saudi Arabia
}

\begin{tabular}{|c|c|}
\hline ARTICLE INFO & ABSTRACT \\
\hline Published Online: & Introduced the homological algebra and presented some interesting basic properties of the notion.In \\
\hline 19 January 2022 & this paper we extend the above notion to homology groups and tried to proof the some similar basic \\
\hline Corresponding Author: & properties of the topological homolog groups. We also studied more about the random graph groups of \\
\hline Abdel Radi Abdel & the homology order to find necessary and sufficient conditions for which the hematology is discrete. \\
\hline $\begin{array}{l}\text { Rahman Abdel Gadir } \\
\text { Abdel Rahman }\end{array}$ & $\begin{array}{l}\text { We followed the analytical induction mathematical method and we found that studying homology } \\
\text { groups may be more important than cohomology groups. }\end{array}$ \\
\hline \multicolumn{2}{|c|}{$\begin{array}{l}\text { KEYWORDS: Homology - Cohomological - Dimension -Graph - Random- Graph Groups -Sub Graph -Maximal - Complex - } \\
\text { Homology Groups -Shine - Image }\end{array}$} \\
\hline
\end{tabular}

\section{INTRODUCTION}

Algebra homology is twentieth century field of mathematics that can trace its origins and connection and homology is one of the main idea of algebraic topology. Algebra's topology is one of the most important creation in mathematics which uses algebra tools to study homological groups. The most important of this invariants are homology groups, cohomology groups. The goal of this paper is to acquire uses study of some classes of algebraic homology (some underline geometry notation). Homology groups, graph and topology theory and cohomological diminution of random graph groups.

\section{SIMPLICIAL COMPLEXES AND HOMOLOGY GROUPS}

For now we shall deal mainly with dimensions 2 and 3. Animpticit exercise throughout this is to generalize all the definitions and proof to higher dimensions.

\section{Definition (2.1):}

An oriented $0-$ simplex is apoint P: Anoriented 1- simplex is a directed line segment $P_{1} P_{2}$. An oriented $2=$ simplex is triangle $P_{1} P_{2} P_{3}$ with a prescribed order.

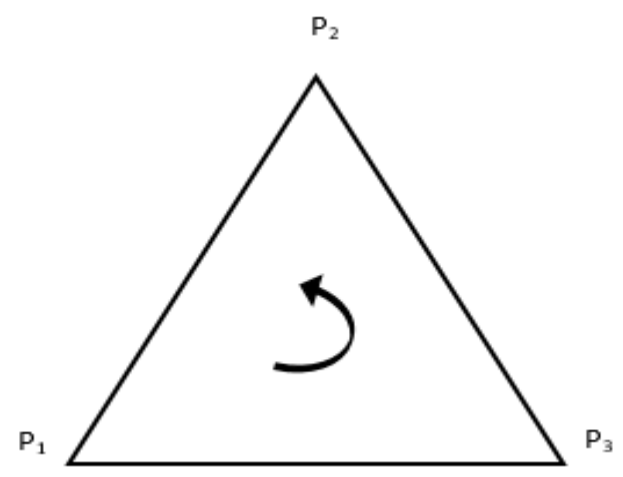

Figure No.2.1 An Oriented 2-Simplex 
Notice that the simplex of opposite orientations is (defined to be) the negative of the simplex:

$$
\begin{aligned}
& \text { 1. } P_{1} P_{2}=-P_{2} P_{1} \neq P_{2} P_{1} \\
& \text { 2. } P_{1} P_{2} P_{3}=P_{2} P_{3} P_{1}=P_{3} P_{1} P_{2}=-P_{1} P_{2} P_{3}=-P_{2} P_{3} P_{1}=-P_{2} P_{1} P_{3}
\end{aligned}
$$

An oriented 3 - simplex is a tetrahedron . $P_{1} P_{2} P_{3} P_{4}$ with a prescribed orientation.

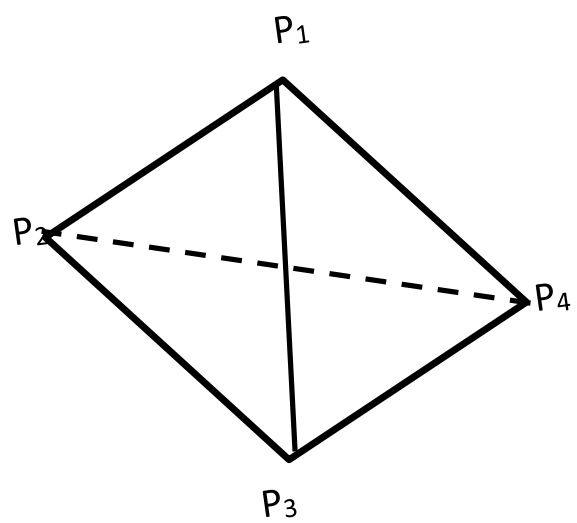

Figure No. 2:2 An Oriented 3-Simplex

Notice that 2- Simplexes:

$$
P_{i} P_{j} P_{k}=\operatorname{sign}\left[\begin{array}{lll}
1 & 2 & 3 \\
i & j & k
\end{array}\right] P_{1} P_{2} P_{3}
$$

And this extend to higher dimensions.

The equation $\partial^{2}=0$.

$$
P_{i} P_{j} P_{k} P_{e}=\operatorname{sign}\left[\begin{array}{llll}
1 & 2 & 3 & 4 \\
i & j & k & e
\end{array}\right] P_{1} P_{2} P_{3} P_{4}
$$

Theorem 2.2 : Let $\mathrm{K}$ be a simpelical complex. Then the homomorphism

Is trivial that is, $\partial^{2}=0$

$$
\partial n_{1}=\partial n: G_{n}(k) \rightarrow G_{n}-2(k)
$$

Proof: first we show that is enough to check this on simplexes, since these generate $C_{n}$

Then simply calculate: for example in dimension 2 we have

$$
\partial_{1}\left(\partial_{2}\left(P_{1} P_{2} P_{3}\right)\right)=\partial_{1}\left(P_{2} P_{3}-P_{1} P_{3}+P_{1} P_{2}\right)=\left(P_{3}-P_{2}\right)-\left(P_{3}-P_{1}\right)+\left(P_{2}-P_{1}\right)=0
$$

Corollary 2.3: $B_{n}(\mathrm{k})=\partial_{n+1}(n+1(k))$ is a sub group of $\mathrm{Zn}(\mathrm{k})=\operatorname{ker}\left(\partial_{n}\right)$.[5]

\section{HOMOLOGICAL ALGEBRA}

We compute homology groups, and to construct the homology groups of pair of spaces. These turn out to be stronger invariants from chain groups.

\section{Definition 3.1:}

A chain complex $\langle\mathrm{A}, \partial\rangle$ is a doubly infinite sequence $A=\left\{\ldots, A_{2}, A_{1}, A_{0}, A_{-1}, A_{-2}, \ldots.\right\}$

Of Belgian groups $\mathrm{A}_{\mathrm{k}}$, together with A collection $\partial=\left\{\partial_{k} \mid \mathrm{k} \in \mathrm{z}\right\} \quad$ Of homeomorphisms such that $\partial_{k}: A_{k} \rightarrow A_{k-1}$ And $\quad \partial_{k} \partial_{k-1}=0$

For braving we shall sometimes denote the chain complex by $\langle$ A, $\partial\rangle$ A. in a chain complex, it is clear that the image of $\partial_{k}$ is a subgroups of the kernel of $\partial_{k-1}$

\section{Definition 3.2:}

If $\mathrm{A}$ is a chain complex, then the kernel $z_{k}(A)$ of $\partial_{k}$ is the group of $\mathrm{K}$ - cycles, and the image $B_{k}(A)=\partial_{k+1}\left(A_{k+1}\right)$

Is the group of $\mathrm{K}$ - boundaries, the factor group

$H_{k}(A)=Z_{k}(A) / B_{k}(A)$

Is the $\mathrm{K}$ the homology group of $\mathrm{A}$. [11]

\section{Example 3.3:}


Let $\mathrm{X}$ be the one - dimensional simplicial complex shown in figure 3.3

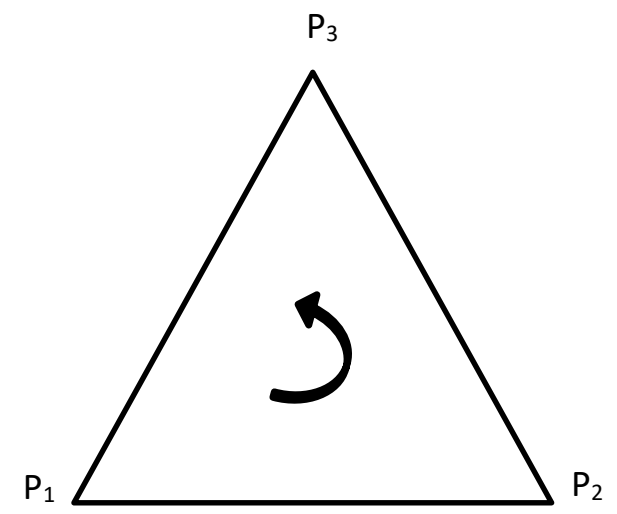

Figure No. 3:3: The Simplicial Complex X

And let y be the sub complex consisting of the edge $P_{1} P_{3}$

We know that

$$
H_{1}(x) \cong Z
$$

Geometrically, shrinking $P_{2} P_{3}$ to a point collapses the rim of the triangle, as shown in figure 3.4

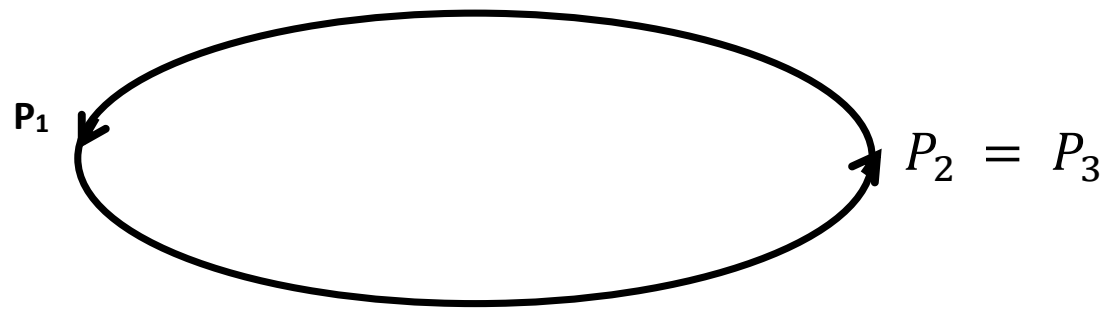

Figure No. 3.4 The Simplicial Complex X with Edge $P_{2} P_{3}$ Shrunk to a Point

\section{Theorem 3.4The Chain Complex(3.1) is Exact:}

The exact sequence is called the exact homology sequence of the pair $\left(\mathrm{A}, \mathrm{A}^{*}\right)$. If the chain complexes arise from a pair of topological spaces. In this case, we obtain for subcomplex $\mathrm{L}$ of the simplicial complex K,

$\stackrel{j * 1}{\rightarrow} \rightarrow H_{1}(K L) \stackrel{j * 1}{\rightarrow} \rightarrow H_{0}(k) \stackrel{j * 0}{\rightarrow} H_{0}(k, L) \rightarrow 0$.[9]

$$
\begin{aligned}
\stackrel{\partial * k+1}{\longrightarrow} \rightarrow H_{k}(L) \stackrel{i * k}{\rightarrow} \rightarrow H_{k}(k) \stackrel{j * k}{\rightarrow} \rightarrow H_{k}(k, L) \\
\stackrel{\partial * k}{\rightarrow} \rightarrow H_{k-1}(L) \stackrel{i * k-1}{\longrightarrow} \rightarrow H_{k-1}(k) \stackrel{i * k-1}{\longrightarrow} \rightarrow H_{k-1}(k, L) \stackrel{i * k-1}{\longrightarrow}
\end{aligned}
$$

\section{Graph:}

Example 4.1 Two important families graphs are the complete graphs and the complete bipartite graphs. The complete graphs on $n$ vertices is the graph $\mathrm{K}_{\mathrm{n}}$ that has $\mathrm{n}$ vertices and a collection of edges such that each pair of distinct vertices is joined by a single edge (see figure 4.1)

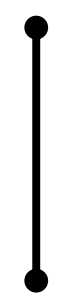

$K_{2}$

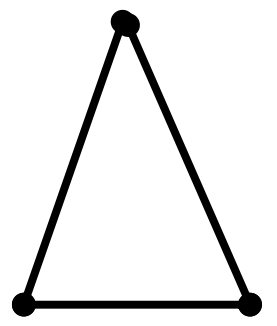

$\mathrm{K}_{3}$

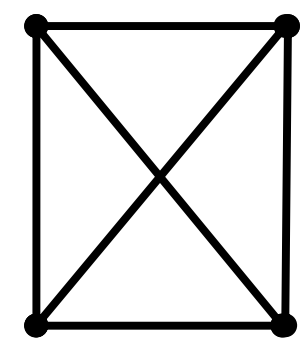

$\mathrm{K}_{4}$

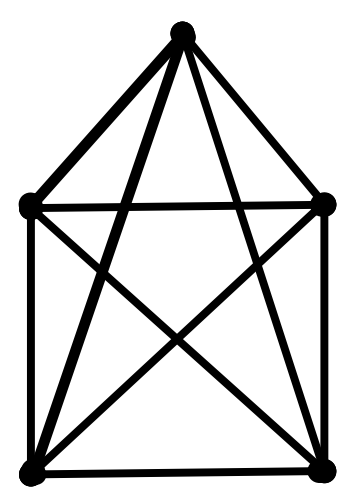

$\mathrm{K}_{5}$

Figure N0. 4.1: The Complete Graphs $\mathrm{K}_{2}, \mathrm{~K}_{3}, \mathrm{~K}_{4}, \mathrm{~K}_{5}$ [8] 


\section{Definition 4.2:}

The complete bipartite graph $K_{m n}$ is a graph having mtn vertices, respectively, such that:

i. Each edge joined a vertex in $V_{m}$ to a vertex in $V_{n}$,

ii. Each pair of vertices $V \in V_{m}$ and $V \in V_{n}$ is joined by a single edge.

Examples 4.3 Of Complete Bipartite Graphs are Shown in Figure 4.3

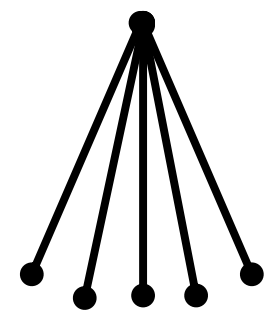

$\mathbf{K}_{1.5}$

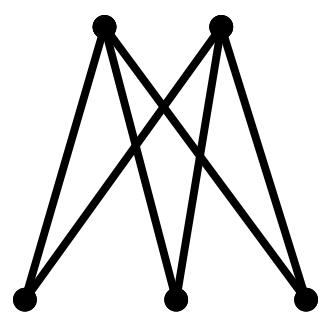

$\mathbf{K}_{2.3}$

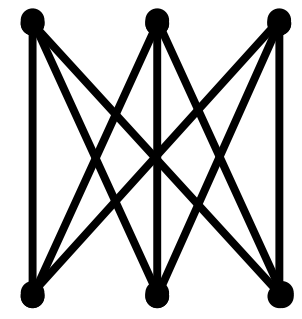

$K_{3.3}$

Figure N0. 4.3: The Complete Bipartite Graph $\mathrm{K}_{1.5}, \mathrm{~K}_{2.3}, \mathrm{~K}_{3.3}[\varepsilon$,

Theorem 4.4: Let $G$ and $G^{y}$ be graphs with vertex sets $V_{G}$ and $V_{C}$ respectively if there is a homeomorphism $h: G \rightarrow G^{r}$ that's maps $V_{G}$ bigectvely to $V_{G}$ then $\mathrm{G}$ and $\mathrm{G}$ are isomorphic and the function $h_{v} V_{G} \rightarrow V_{G r}$ defined by $h v(V)$ is a graph isomorphism.

Proof:

If we drop the assumption in this theorem that $\mathrm{h}$ maps the vertex set of $\mathrm{G}$ bigectvely to the vertex set of $\mathrm{G}$, then it does not necessarily follow that $\mathrm{G}$ and $G^{-}$are homeomorphic but not isomorphic.
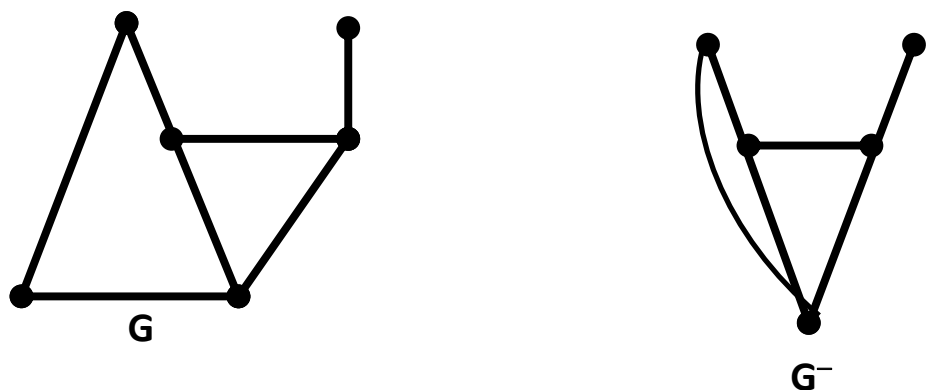

Figure No. 4.4 Graphs $\mathrm{G}$ and $G^{-}$are Hometmorphic but not Isomorphic [9]

\section{5. $L^{2}$ - COHAMOLOGY}

Definition 5.1 We define the cellular

$$
L^{2} \text { - cohamology by } H_{(2)}^{p}(\bar{X} . \Gamma)=\operatorname{ker}\left(d^{p}\right) / \overline{\imath m}\left(d^{p-1}\right)
$$

We have affodge decomposition

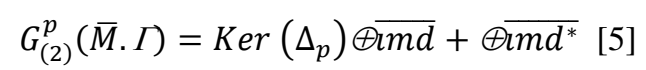

Theorem 5.2 $L^{2}$ - cohomology and in particular $L^{2}$ - Bett numbers have the following basic properties. Here, let $\bar{X}$ be a normal covering of a finite GW- complex X with covering group $\Gamma$.

1. Assume $\Gamma$ is finite, then $\bar{X}$ is itself a finite GW- complex and it (ordinary) Bett number $b_{(2)}^{p}(\bar{X} . \Gamma)=\frac{1}{|\Gamma|} b^{p}(\bar{X})$

\section{Proof:}

If $\Gamma$ is finite. all Hebert spaces in question are finite dimensional.

Consequently, im (d) is antomatically closed, and there is no difference between $L^{2}$ - cohamology and ordinary cohomology with complex coefficients of $\bar{X}$ in particular.

$$
b^{p}(\bar{X})=\operatorname{dim}_{c} H_{(2)}^{p}(\bar{X}, I)=\operatorname{dim}_{\Gamma}\left(H_{(2)}^{p}(\bar{X} . \Gamma)\right)=\mid \Gamma \cdot b_{(2)}^{p}(\bar{X} . \Gamma)[8]
$$

Proposition 5.3: For $\mathrm{G}$ afinite group, the homology $H *(G, m) . *>0$, is totsion of exponent $|G|$ for any G- module M. If $\mathrm{M}=\mathrm{Z}$ with travial G- action, we write $H *(G)=H *(G, Z)$ here are some useful properties:

1. $H_{1}(G)=G /|G, G|$.

2.If $G=F / R$ were $\mathrm{F}$ is a free group and $\mathrm{R}$ is normal subgroup of $\mathrm{F}$ then $H_{2}(G)=R \cap[F, F] /[F, R]$ (Hopf.sformular)

3.If $G=B_{1} * B_{2}$ is the amalgamated free product of $B_{1}$ and $B_{2}$ over a common subgroup A. then there is an exact sequence 


$$
\begin{aligned}
\ldots . . \rightarrow & H_{i}\left(B_{1}\right) \oplus H_{i}\left(B_{2}\right) \rightarrow H_{i}(G) \rightarrow H_{i-1}(A) \rightarrow n \\
& 4 . H_{i}(G X Z)=H_{i}(G) \oplus H_{i-1}(G)[12]
\end{aligned}
$$

\section{COHOMOLOGY}

\section{Definition 6.1:}

Given a cohomology filtration $\mathrm{F}=\mathrm{F}^{\prime \prime}$ of an R-module A the associated graded module is the graded, R-module denoted by $\mathrm{Gr}$ $(\mathrm{A}, \mathrm{F})$ and defined by

$$
\operatorname{Gr}(A, F)^{p} \frac{F^{p}}{F^{p+1}}[10]
$$

\section{Definition 6.2:}

A straight forward verification shows that $\mathrm{b} 2=0$ Thus, the Hoch child differential defines the two complexes :

$$
\begin{aligned}
0 & \rightarrow C_{\lambda}^{0}(A) \stackrel{b}{\rightarrow} C_{\lambda}^{1}(A) \stackrel{b}{\rightarrow} C_{\lambda}^{1}(A) \stackrel{b}{\rightarrow} \ldots .(C .10) \\
0 & \rightarrow C_{\lambda}^{0}(A) \stackrel{b}{\rightarrow} C_{\lambda}^{1}(A) \stackrel{b}{\rightarrow} C_{\lambda}^{1}(A) \stackrel{b}{\rightarrow} \ldots .(C .11)
\end{aligned}
$$

The cohomology group of the complexes (C.10) and (C.11) are called the cyclic cohomology and the Hochschild home age groups of the complexescc-101 and can cohomology. respectively, of the algebra A and are denoted by

$$
\begin{gathered}
H c^{z}(A)=Z_{\lambda}^{n}(A) / B_{\lambda}^{n}(A) \\
H c^{z}(A)=Z_{\lambda}^{n}(A) / B_{\lambda}^{n}(A)[7]
\end{gathered}
$$

\section{Definition 6.3:}

A cohomology theory for Paws of Spaces with values in the category of R-modules consists of a family (h"Inez) of contravariantfunctors

$$
h^{n}: T o P(2) \rightarrow R-M o D
$$

and a family $\delta^{n} \mid$ nez of natural transformations

$$
\delta^{n}: h^{n-1} \text { ok } \rightarrow h^{n}[5]
$$

\section{Definition 6.4:}

Let $\bar{T}=\Omega^{n} \rightarrow c$ be acontinuous Linear functional possessing the cyclic invariance property:

$$
\bar{T} w_{1} w_{2}=(-1)^{k i} \bar{T} w_{2} w_{1}, \quad w_{1} \in \Omega^{k}, \bar{T}=w_{2} \in \Omega^{i}, k+i=n
$$

Each functional of this form will be called a graded track of degree non $\Omega$. [5]

\section{Definition 6.5:}

Given a brigaded cohomology spectral sequence $E_{r}^{p q}, d r$ and aggraded R-module $A^{*}$ we say the spectral Sequence converges to $A^{*}$ and write

If:

$$
E_{2}^{p q} \Rightarrow A^{p+q}
$$

1. For each (p, q) there exists an $r_{0}$ so that $d r: E_{r}^{p-r, q+r-1} \rightarrow E_{r}^{p q}$ is 0 for all $r \geq r_{0}$, in particular there is an injection $E_{r+1}^{p, q} \rightarrow$ $E_{r, q}^{p}$ for all $r \geq r_{0}$.

2. there is a convergent filtration of $A^{*}$, so that for each $\mathrm{n}$, thelimit $E_{\infty}^{p, q}=\cap_{r p \geq r 0} E_{r}^{p, q}$ is isomorphic to the associated graded $\Rightarrow \operatorname{Gr}\left(A^{*}\right)$. [6]

\section{Definition 6.6:}

If $\mathrm{M}$ is an $\mathrm{A}$-module and $\mathrm{p} \geq 0$, then the path exterior power of $\mathrm{M}$, denoted by $\Lambda^{p} M$, is the abelian group with the following Presentation.

Generators: $A x M x, \ldots, x M$ (P factorsm).

Relations: For all $a, a^{\prime} \in A$ and $m_{i}, m_{i} \in M$,

$$
\left(a, m_{i}, \ldots, m_{i}+m_{i}, \ldots, m_{p}\right)=\left(a, m_{i}, \ldots, m_{i}, \ldots, m_{p}\right)+\left(a, m_{i}, \ldots, m_{i}^{\prime}, \ldots, m_{p}\right)
$$

For all i,

For all i

$$
\left(a+a^{\prime}, m_{i}, \ldots, m_{p}\right)=\left(a, m_{i}, \ldots, m_{p}\right)\left(a a^{\prime}, m_{i}, \ldots, m_{i}, \ldots, m_{p}\right)=\left(a, m_{i}, \ldots, a^{\prime m_{i}}, \ldots, m_{p}\right)
$$

$\left(a, m_{i}, \ldots, m_{p}\right)=0$ if $m_{i}=m_{j}$ for some $i \neq j$

$\Lambda^{1} M \cong M[11]$

$$
\text { if } p=0 \text {, then } \Lambda^{0} M=A \text {, andif } p=1 \text {, then }
$$

\section{Definition 6.7:}

For a based space $\mathrm{x}$, we define the reduced cohomology of 


$$
\begin{gathered}
\mathrm{x} \text { to be } \\
E^{-q}(x)=E^{q}(x, *)
\end{gathered}
$$

These results a direct Sussi decomposition:

$$
E^{*}(x) \cong E^{*}(x) \oplus E^{*}(*)
$$

That is natural with respect to based maps for $* \in A c x$, the Summand $E^{*}(A)$ maps isomorphic ally under the maps $E^{*}(x) \rightarrow E^{*}(A)$, and the exactness axiom implies that there is a reduced long exact sequence

$$
\ldots \rightarrow E^{-q-1}(A) \stackrel{\delta}{\rightarrow} E^{q}(x, A) \rightarrow E^{-2}(x) \rightarrow E^{-2}(A) \rightarrow \cdots
$$

The unreduced cohomology groups are recovered as the special cases.

$$
E^{*}(x)=E^{*}\left(x_{1}\right)[10] .
$$

\section{Definition 6.8:}

A cochain complex $\mathrm{G}$ is a z-graded R-module with a graded homogeneous homomorphism of degree $=1$. It is customary to denote the components of a cochain complex by Superscripts $\mathrm{c} 2$ and call the graded homeomorphisms

$d^{q}: c^{q} \rightarrow c^{q+1}[6]$.

\section{RESULTS}

we showed that the discuss of the constant mean Co-homological dimension of random graph groups and we found the following results:

1. We showed that the homology group gives accurate and high-speed results.

2. The ability of the graph or the diagram to any group through the homology and Possibility of calculating the constant mean cohomological dimension of random graph a group.

\section{CONCLUSION}

we calculated the constant mean co-Homological dimension of random graph groups, we also discuss many concepts of Simplicial complexes and Homology groups Homological algebra, basic theories of graph and Homology.

\section{REFERENCES}

1. A.E. Costa. M. Farber. T. Kappeler, Topology of random 2-compleres, preprint 2010, arXiv:1006.4229.

2. A.E. Costa, M. Farbøf. Topology of random right angled Artin groups, Journal of Topology and Analysis, 3(2011). 28987.

3. Bollubis, Random Graphs. Second edition, Cambridge University Press, 2008. Cambridge Stud. Adv. Math., 73, Cambridge, 2001.

4. D. Cohen. M. Farber. T. Kappeier, The homolopical dimension of random 2-compleres, preprint 2010. arXiv:1005.3383v1.

5. D. Cohen. A.E. Costa. M. Farber, T. Kappeler, Topology of random 2-complexes, to appear in Discrete and Computational Geometry.

6. E Babson. C. Hoffman, M. Kahle, The fundamental group of random 2-compleres, J. Amer. Math. Soc. 24 (2011), 1-28.

7. L. Aronsitan., N. Linial, T. Luczak, R. Meshulam, Vanishing of the top homology of a random complex. arXiv:1010.1400.

8. N. Alon. J. Spencer. The Probabilistic Method, Third edition. Wiley-Intersci. Ser. Discrete Math. Optim.. John Wiley \& Sons, Inc., Hoboken, NJ. 2008.

9. R. Charncy, An introduction to right-angled Artin groups, Geom. Dedicata 125 (2007), 141-158.

10. R. Charnev. M. Farber, Random groups arising as graph products, arXiv:1006.3378.

11. R. Charney, K. Vogtmann, Finitepless properties of automorphism groups of right-angled Artin groups. Bulletin LMS (2009); doł: 10.1112/blms/bdn 108.

12. R. Charney, K. Vogtrany. Subgroups and quotients of automorphism groups of RAAGS. arXiv:0909.2444. 